\title{
Collagen fleeces do not improve colonic anastomotic strength but increase bowel obstructions in an experimental rat model
}

\author{
Marc H. Schreinemacher • Johanne G. Bloemen • \\ Stijn J. van der Heijden • Marion J. Gijbels • \\ Cornelis H. Dejong $•$ Nicole D. Bouvy
}

Accepted: 1 February 2011 / Published online: 23 February 2011

(C) The Author(s) 2011. This article is published with open access at Springerlink.com

\begin{abstract}
Purpose To investigate whether a collagen fleece kept in place by fibrin glue might seal off a colorectal anastomosis, provide reinforcement, and subsequently improve anastomotic healing.

Methods Wistar rats underwent a 1-cm left-sided colonic resection followed by a 4 -suture end-to-end anastomosis. They were then randomly assigned to one of three treatment groups: no additional intervention (control, $n=20$ ), the anastomosis covered with fibrin glue (fibrin glue, $n=20$ ), the anastomosis covered with a collagen fleece, kept in place with fibrin glue (collagen fleece, $n=21$ ). At either 3 or 7 days follow-up, anastomotic bursting pressure was measured and tissue was obtained for histology and collagen content assessment after which animals were sacrificed.

Results Three rats in the control (15\%), three in the fibrin glue $(15 \%)$, and one in the collagen group $(4.8 \%)$ died due to anastomotic complications $(P=0.497)$. Anastomotic bursting pressures were not significantly different between groups at 3 and 7 days follow-up $(P=0.659$ and $P=0.427$, respectively). However, bowel obstructions occurred sig-
\end{abstract}

M. H. Schreinemacher $(\triangle) \cdot$ J. G. Bloemen •

S. J. van der Heijden - C. H. Dejong • N. D. Bouvy

Department of General Surgery,

Maastricht University Medical Centre,

P.O. Box 5800, 6200 Maastricht, The Netherlands

e-mail: m.schreinemacher@gmail.com

M. J. Gijbels

Department of Molecular Genetics,

Maastricht University Medical Centre,

Maastricht, The Netherlands

M. J. Gijbels

Department of Pathology, Maastricht University Medical Centre,

Maastricht, The Netherlands nificantly more often in the collagen group compared to the control group ( $14 / 21$ vs. $3 / 20, P=0.003)$. Collagen contents were not different between groups, but histology showed a more severe inflammation in the collagen group compared to the other groups at both 3 and 7 days follow-up.

Conclusions A collagen fleece kept in place by fibrin glue does not improve healing of colonic anastomoses in rats. Moreover, this technique induces significantly more bowel obstructions in rats, warranting further study before being translated to a clinical setting.

Keywords Anastomotic dehiscence - Rat · Fibrin glue Collagen fleece $\cdot$ Bursting pressure

\section{Introduction}

Clinically significant anastomotic leakages remain a major complication of colorectal surgery. Patients suffering from an anastomotic leakage undergo more radiological interventions, reoperations, and often stay in ICU for prolonged periods of time. Moreover, they experience higher rates of mortality, lower quality of life, and higher rates of local recurrence in malignant disease [1-5].

In the past, there have been many studies - both animal and clinical - on anastomotic leakage prevention. A wide variety of interventions have been investigated aiming at adequate oxygenation and perfusion of the anastomotic region, stimulation of angiogenesis, administration of growth factors, and inhibition of matrix metalloproteinases [6-9]. Other ways to prevent leakages included a more mechanical approach with topically applied sealants and membranes such as fibrin glue and collagen fleeces, respectively. Fibrin sealants are human- and animalderived tissue adhesive agents that can be used to enhance 
hemostasis and tissue sealing starting directly after surgery [10]. Collagen fleeces are mainly animal-derived collagen matrices that have been found to assist in the healing of gastrointestinal wounds [11].

Up to now, most studies using this approach of sealantor fleece-reinforced anastomoses concluded there was only limited or no significant improvement in anastomotic healing [12-14]. In addition, experimental anastomoses were constructed with up to 20 sutures leading to clinical leakage rates close to zero. In such cases, this results in extremely small interstices which is not comparable with the human situation in which leaks often result from larger interstices. Particularly in these cases, the sealing effect of fibrin glue together with the reinforcement of a collagen fleece could theoretically improve anastomotic healing.

We therefore hypothesized that a collagen fleece secured to the anastomosis with fibrin glue could significantly improve anastomotic healing in a rat model with a 4-suture left-sided colonic anastomosis.

\section{Material and methods}

Male Wistar rats, weighing 300 to $350 \mathrm{~g}$ were housed and cared for at the Central Animal Facilities of Maastricht University with food and water ad libitum. The experimental protocol complied with the Dutch Animal Experimentation Act and was approved by the Animal Ethics Committee of Maastricht University. After construction of the anastomosis, we assigned rats to one of three treatment groups following randomization with sealed envelopes: no additional intervention (control, $n=20$ ), covering the anastomosis with fibrin glue (fibrin glue, $n=20$ ), covering the anastomosis with a collagen fleece, kept in place with fibrin glue (collagen fleece, $n=21$ ). At either 3 or 7 days follow-up, half of each group was sacrificed $(n=10$ per group at each time point, except $n=11$ for 7 days collagen fleece).

\section{Surgical procedure}

Anastomoses were constructed as described earlier [15]. In brief, rats received $0.1 \mathrm{mg} / \mathrm{kg}$ of buprenorphine subcutaneously for analgesia. Anesthesia was induced with isoflurane $5 \%$ and maintained with isoflurane 2.0 $2.5 \%$. Rats were not fastened prior to surgery. The abdomen was shaved and the skin disinfected with $2 \%$ iodine. Following a $4-\mathrm{cm}$ midline incision, a small segment of the left colon, extending from 2 to $1 \mathrm{~cm}$ above the peritoneal reflection was resected. An end-to-end anastomosis was constructed with four evenly distributed, interrupted polypropylene $6 \times 0$ sutures. In case of applying fibrin glue, Tisseel Duo (Baxter, Vienna, Austria) at $37^{\circ} \mathrm{C}$ was sprayed all around the anastomosis using the Easyspray (Baxter) at a pressure of 1.5 bar. In case of applying a collagen fleece, a single layer of Tissufleece (Baxter) extending $6 \mathrm{~mm}$ to each side of the anastomosis was wrapped around the anastomosis after applying fibrin glue. Then, the peritoneum and fascia were closed with a running polyglactin $4 \times 0$ suture. The skin was closed intracutaneously with a running polyglecaprone $4 \times 0$ suture. On the first day postoperatively, a single shot of $0.1 \mathrm{mg} / \mathrm{kg}$ buprenorphine was administered to all rats.

\section{Bowel obstruction}

Bowel obstructions were defined as a colon distended more than two times its normal diameter proximal to the anastomosis and tapering to a normal diameter distal to the anastomosis.

\section{Bursting pressure}

At either 3 or 7 days, rats were anesthetized as described above. The midline laparotomy was opened and the anastomotic region identified without any manipulation of adhering tissue. The colon was transected approximately $3 \mathrm{~cm}$ proximal to the anastomosis, followed by insertion of the tube of a manometer (OJB Industries, Groningen, The Netherlands) which was secured with a suture. Distally from the anastomosis, a clamp was placed just above the peritoneal reflection. Subsequently, the abdomen was filled with $0.9 \% \mathrm{NaCl}$ solution at $37^{\circ} \mathrm{C}$ until full submersion of the anastomotic region. Then, the air pressure was gradually increased. The bursting pressure was defined as the maximum pressure read out on the manometer before bubbles appeared. Thereafter, rats were sacrificed by opening the diaphragm and cutting the ventricles. The anastomotic region was resected and fixed in $4.0 \%$ formaldehyde for $24 \mathrm{~h}$ after which the specimens were embedded in paraffin.

\section{Histological assessment}

Slices $4 \mu \mathrm{m}$ thick were stained with hematoxylin and eosin (H\&E) using standard histological techniques. Tissue sections were also stained for collagen using picro-sirius red. For this purpose, sections were deparaffinized in xylene, rehydrated in graded ethanol to distilled water, and subsequently pretreated with $0.2 \%$ phosphomolybdic acid for $5 \mathrm{~min}$. Then, sections were stained for $90 \mathrm{~min}$ in $0.1 \%$ solution of Sirius Red in saturated aqueous picric acid followed by a 2-min wash in $0.01 \mathrm{~N}$ HCL. Hereafter, sections were dehydrated in ethanol and mounted in entallan. Pictures of the anastomotic and perianastomotic 
regions were taken at a magnification of $\times 200$ using cross polarization light microscopy (Leica DM5000B, Leica Microsystems, Heerbrugg, Switzerland). The collagen content in the anastomotic and perianastomotic region was quantified as a percentage of total tissue surface and expressed as relative collagen area using the Qwin morphometry system (Leica QWin V3.5.1, Leica Microsystems). Furthermore, maturity of collagen was estimated by calculating the red versus green ratio, where red indicates thick, more mature collagen fibers and green indicates the thinner, immature collagen fibers. All histological assessments were performed by an experienced pathologist, blinded to the study groups.

\section{Statistical analysis}

All data are expressed as means \pm standard error of means (SEM). Loss of body weight, bursting pressures, and collagen ratios were compared using one-way ANOVA and paired samples $t$ test. Mortality and bowel obstructions were analyzed using Chi square and Fisher's exact tests. A Bonferroni corrected $P$ value $<0.050$ was considered statistically significant. All analyses were performed using SPSS version 15.0 (SPSS, Chicago, Illinois, USA).

\section{Results}

Rats lost an average of $8.8 \pm 0.8 \%$ of initial body weight during the first 4 days after surgery $(P=0.107$ between groups), regaining weight from day 5 . Three rats in the control group ( $15 \%$; one on day 2 , two on day 3$)$, three in the fibrin glue group (15\%; on days 1,3 , and 5$)$, and one in the group with a collagen fleece $(4.8 \%$; on day 3$)$ died before the intended time of sacrifice $(P=0.497)$. All but two of these rats showed signs of fecal peritonitis with dehiscence of the anastomosis at autopsy. The other two (one with fibrin glue and one with a collagen fleece) showed pus on the anastomosis accompanied with a mild peritonitis. Minimal to moderate adhesions between the anastomotic region and peritesticular fat or seminal vesicles were found in most rats. Implanted collagen fleeces were still in place at 3 days and partly absorbed at 7 days follow-up.

\section{Bursting pressure}

Bursting pressures were not significantly different among control, fibrin glue, and collagen fleece groups at 3 days follow-up $(60.5 \pm 11.3,67.9 \pm 12.6$, and $50.7 \pm 15.5 \mathrm{mmHg}$,

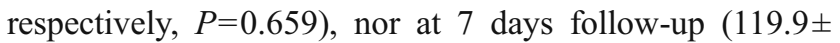
$27.9,167.7 \pm 32.0$, and $114.8 \pm 27.5 \mathrm{mmHg}$, respectively, $P=0.427$ ) (Fig. 1). In general, a significant increase in

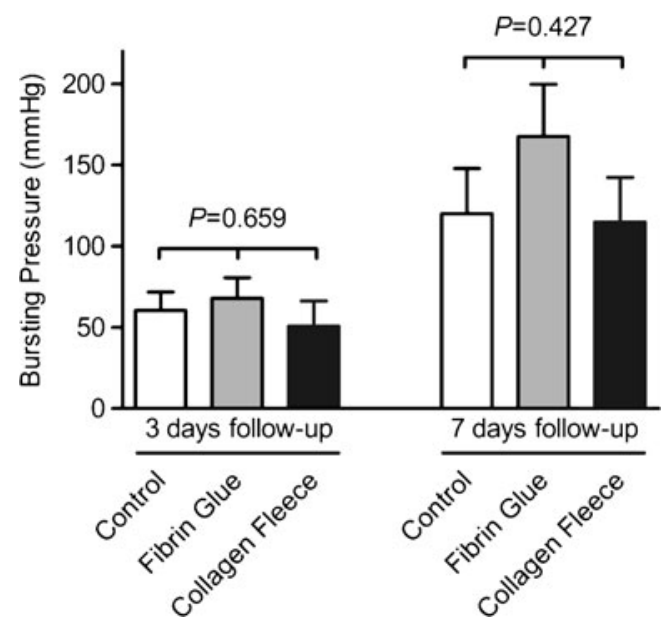

Fig. 1 Bursting pressures were measured by intraluminally increasing the air pressure while the anastomotic area was submerged. The maximum pressure before bubbles appeared was regarded the bursting pressure. Both at 3 and 7 days follow-up, there were no significant differences between groups. All means (SEM)

bursting pressures occurred between 3 and 7 days follow-up (59.7 \pm 41.1 vs. $130.4 \pm 86.4 \mathrm{mmHg}, P<0.001)$.

Bowel obstruction

Rats with a collagen fleece showed a significantly higher incidence of bowel obstructions than the control group (14/21 vs. 3/20, $P=0.003)$. Anastomoses covered only with fibrin glue showed intermediate proportions of bowel obstructions, not significantly different from the other groups (Fig. 2). In case of a collagen fleece, the lumen at the anastomosis appeared stiff and smaller than the normal colonic lumen. However, lumena in all groups were open.

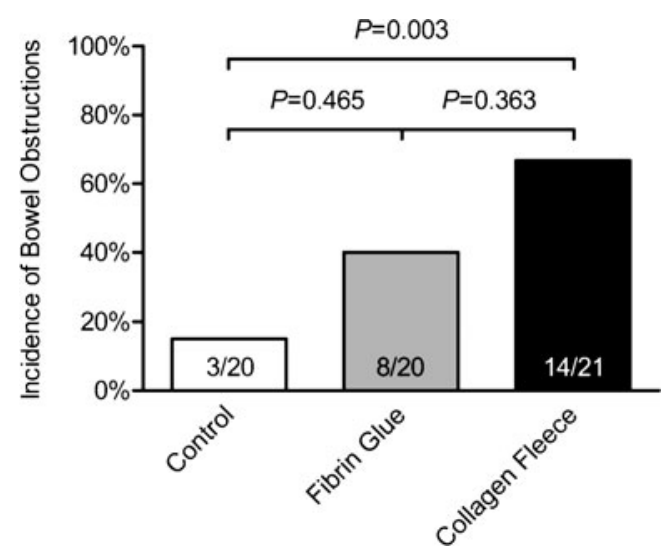

Fig. 2 Bowel obstructions were defined as a colon distended more than two times its normal diameter proximal to the anastomosis and tapering to a normal diameter distal to the anastomosis. Rats with a collagen fleece showed a significantly higher incidence of bowel obstructions than the control group (14/21 vs. $3 / 20, P=0.003)$ 


\section{Collagen content}

No significant differences were observed in the anastomotic regions in terms of relative collagen areas (collagen content expressed relative to total tissue area) and red versus green ratios (collagen maturity) between groups at 3 and 7 days follow-up (Table 1). All groups combined, relative collagen areas at the anastomotic region $(6.1 \pm 1.6 \%)$ were significantly lower than at the perianastomotic region at 3 days follow-up $(15.4 \pm 2.3 \%, P=0.001)$, but equal at 7 days follow-up ( $18.9 \pm 3.3 \%$ vs. $18.3 \pm 3.0 \%, P=0.826)$. This was due to a significant increase in the relative collagen area at the anastomotic region between $3(6.1 \pm 1.6 \%)$ and 7 days follow-up (18.9 $\pm 3.3 \%, P=0.001)$.

\section{Histology}

Anastomotic regions in the control and fibrin glue groups showed a similar acute inflammatory response at 3 days follow-up, characterized by necrosis and granulocyte infiltration (Fig. 3a). Fibrin glue was still microscopically detectable at 3 days follow-up. However, application of a collagen fleece induced a more severe inflammatory reaction with deep necrosis at the fleece-bowel interface (Fig. 3c).

At 7 days follow-up, the control and the fibrin glue group reached a chronic inflammatory state with the formation of granulation tissue and the infiltration of macrophages and lymphocytes (Fig. 3d, e). In addition, the first signs of epithelial cells migrating from the margins of the wound to the defect became apparent (Fig. 3e). However, anastomoses with a collagen fleece still showed a more severe inflammation than the other groups with necrosis and a high density of inflammatory cells, including granulocytes, macrophages, and lymphocytes (Fig. 3f).

\section{Discussion}

In the present study, no beneficial effect of a collagen fleece on anastomotic healing was observed compared to control groups. Bursting pressures and collagen content were not improved, but instead, bowel obstructions and increased inflammation occurred significantly more often in the collagen fleece group.

The return in strength after a gastrointestinal anastomosis is considered to be mainly dependent on the formation of collagen [16]. However, early after the construction of an anastomosis, degradation of the extracellular matrix occurs $[17,18]$. This was observed at 3 days follow-up when a lower collagen content in comparison with the perianastomotic region was found. Thereafter, the remodeling phase begins and the collagen content increases, as observed at day 7. Together with the crosslinking of thin, immature collagen fibers, these processes are generally regarded to improve the anastomotic strength. The strength of the anastomosis can be reliably quantified by examining the bursting pressure [19]. Also, the thicker fibers can be distinguished from the thinner fibers by cross polarization microscopy after picro-sirius red staining. In this study, however, both the bursting pressures as well as the collagen maturation were not improved following fibrin glue and collagen fleece application to the anastomoses.

Besides the absence of any improvements in anastomotic strength, bowel obstructions occurred significantly more often in the collagen fleece group. Although unexpected, other experimental studies have confirmed the occurrence of obstructions after wrapping a fleece of collagen or dura mater around a bowel anastomosis [20, 21]. The exact mechanism remains unclear, but shrinkage of the collagen fleece can be severe and could mechanically impair normal passage of stool, especially in the colon [22]. Additionally, the external constriction by the fleece together with the observed increased inflammatory reaction might induce a more pronounced inward bulging of the edematous colonic wall. A proposed mechanism with increased fibrosis is unlikely since collagen contents did not differ significantly between groups in this study [20]. Yet, it must be noted that this study only displays the results at 3 and 7 days follow-up. After full resolution of the collagen fleece, the colonic wall

Table 1 After picro-sirius red staining of the anastomotic region, the relative collagen area was quantified as the percentage of total tissue surface occupied by collagen

\begin{tabular}{|c|c|c|c|c|c|}
\hline & & Control & Fibrin glue & Collagen fleece & $P$ value \\
\hline \multirow[t]{2}{*}{3 days follow-up } & Relative collagen area & $8.5 \pm 2.7 \%$ & $4.4 \pm 3.7 \%$ & $4.9 \pm 2.0 \%$ & 0.518 \\
\hline & Red/green ratio & $1,195.4 \pm 834.0$ & $398.1 \pm 279.2$ & $227.2 \pm 75.3$ & 0.456 \\
\hline \multirow[t]{2}{*}{7 days follow-up } & Relative collagen area & $25.7 \pm 7.8 \%$ & $17.3 \pm 4.0 \%$ & $14.6 \pm 3.3 \%$ & 0.336 \\
\hline & Red/green ratio & $963.0 \pm 468.5$ & $1,904.5 \pm 707.4$ & $1,664.5 \pm 547.2$ & 0.507 \\
\hline
\end{tabular}

Maturity of collagen was estimated by calculating the red versus green ratio where red indicates thick, more mature collagen fibers and green indicates the thinner, immature collagen fibers. No significant differences were observed between groups at either 3 or 7 days follow-up

All measurements in this table are mean (SEM) 

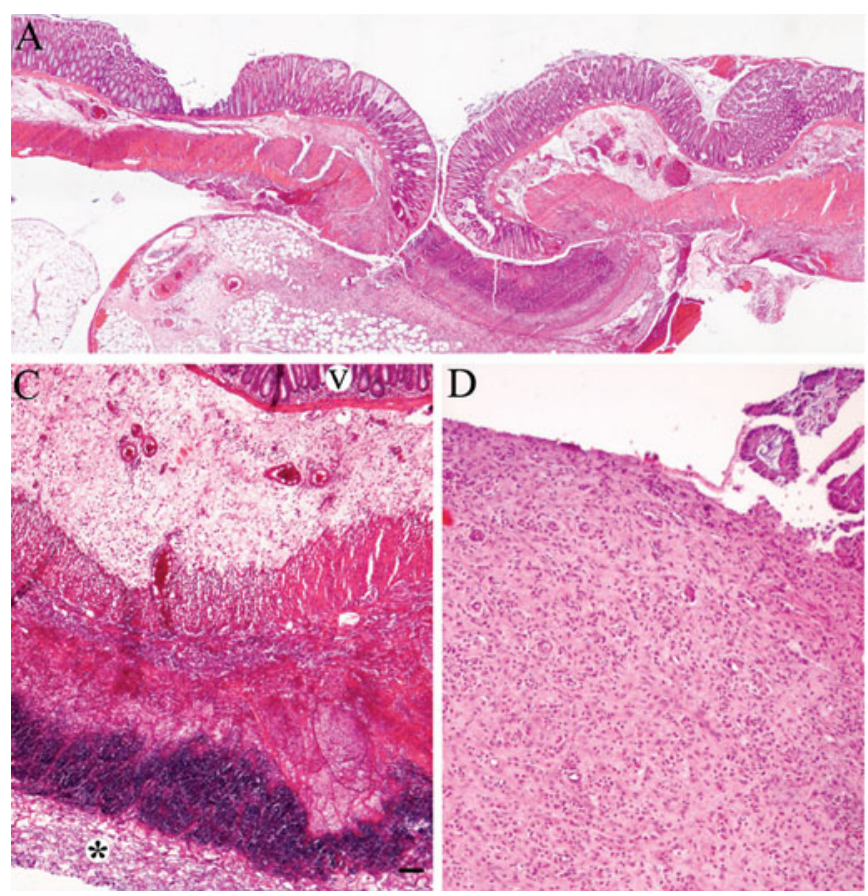

Fig. 3 Light microscopy of anastomoses from different groups. A control anastomosis at 3 and 7 days follow-up (a and $\mathbf{b}$, respectively). At 3 days follow-up, a collagen fleece (asterisk) induced an intense inflammatory reaction (c, $v=$ villi). At 7 days follow-up, the control (d) and the fibrin glue (e) groups showed comparable wound healing at

might remodel further, possibly leading to resolution of the obstruction. Nevertheless, based on our findings, we consider the early period after colonic anastomosis with a collagen fleece at risk for obstruction.

Many sheet-like structures have been tested for their ability to enhance anastomotic healing including collagen fleeces, dura mater fleeces, free peritoneal grafts, polyglycolic acid meshes, and an amniotic membrane [20, 21, 23-25]. Collagen fleeces are derived from the submuscular layer of porcine small bowel or from equine tendons, the latter being the source for the collagen fleeces in this study. At follow-ups longer than 30 days, a collagen fleece seems to be safe, but no significant improvements in bursting pressures have been observed [12]. At shorter follow-up, an impaired healing around the collagen fleece was found in our study and by others [26]. Comparable results were also found after application of dura mater, free peritoneal grafts, and polyglycolic meshes [21, 25]. A possible explanation is the intraluminal confinement of bacteria together with reduced peritoneal contact. Furthermore, one might argue that the avascular and xenograft nature of these sheets is a likely cause for the problems encountered. However, omentoplasties - both vascular and the patient's own-were not able to prevent anastomotic leakages in a large randomized clinical trial either [27]. So far, an amniotic membrane is the only fleece showing potential

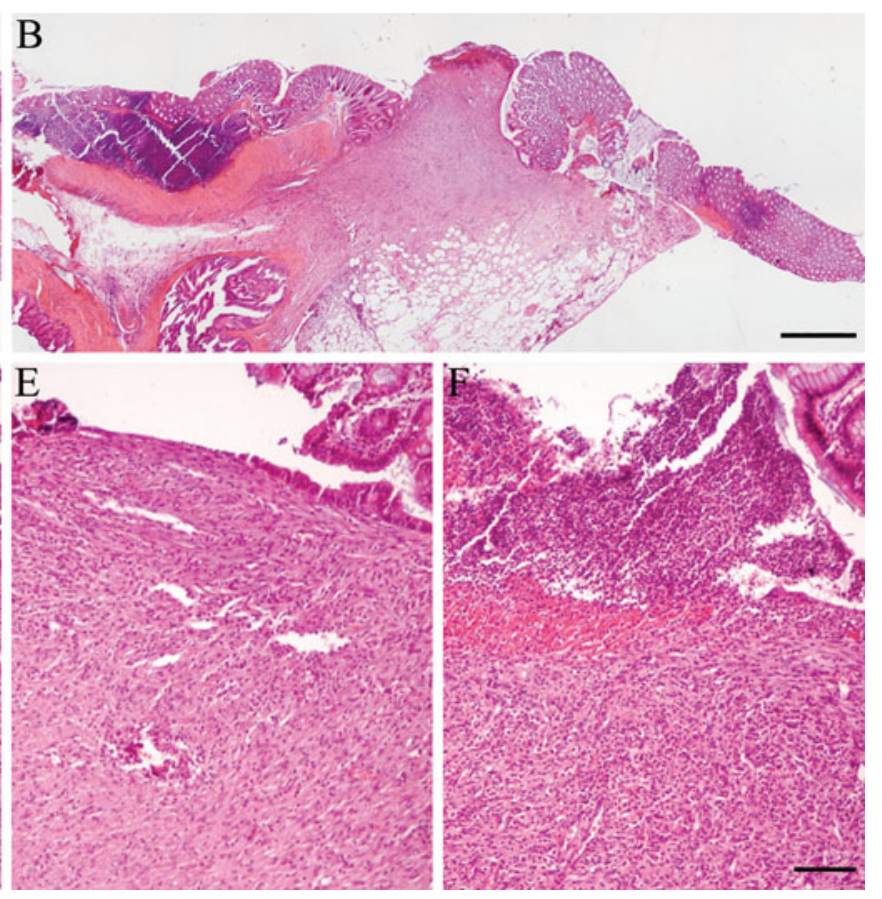

the luminal interface. In the collagen group (f), a more severe inflammation was still present (H\&E staining, bars represent $500 \mu \mathrm{m}$ (a and b, original magnification $\times 40)$ and $100 \mu \mathrm{m}(\mathbf{c}-\mathbf{f}$, original magnification $\times 100)$ )

for anastomotic healing improvement in experimental studies [23, 28].

In this study, no significant differences were found between the fibrin glue group and the control and collagen fleece groups. Other experimental studies have demonstrated an inhibited anastomotic healing due to fibrin glue [14, 29]. In a randomized clinical trial, fibrin glue did not show any improvements on anastomotic leakage after laparoscopic rectal cancer resection [30]. Equally, fibrin glue did not show any improvements in two studies involving laparoscopic gastric bypasses [31, 32]. Yet, leakages after (colo) esophageal anastomoses in children were significantly reduced by fibrin glue [33, 34].

We used four polypropylene sutures in this rat model with colonic anastomoses. We chose unresorbable sutures for this short term follow-up study since we wanted to exclude as much as possible the possible interactions with resorbable materials other than fibrin glue and collagen fleece. Furthermore, apart from a single study in rats, experimental studies typically involved six or more sutures resulting in spontaneous leakage rates equal or close to zero [14]. Our model resulted in a $15 \%$ leakage rate which is comparable with leakage rates in humans. Although this mimics the clinical scenario better, outcomes may be less comparable with other experimental studies. 
Limitations apply to this study. Firstly, we defined bursting pressure as the primary outcome. Bursting pressure has been commonly linked with the occurrence of anastomotic complications such as abscess formation and leakage. Still, these complications can also occur irrespective of improved bursting pressure, for instance after irradiation [35]. In addition, bursting pressures are believed to be associated with collagen content. Yet, growth factorinduced proliferation and migration of cells might improve bursting pressure even without improving collagen content [36]. However, we did not use any radiation or growth factors in this study, limiting this potential source of bias and underlining the applicability of our primary outcome.

Another possible limitation is the definition of an obstruction. In accordance with specific studies on this subject, we chose the clinical finding of a more than twofold distended bowel proximal to the anastomosis. This is in accordance with values found in an experimental obstruction model [37]. Nevertheless, even though a certain degree of constriction occurs at the anastomotic level, it is not clear whether it reaches a critical point at which symptoms occur [12]. With respect to this, the time of follow-up is of interest as well.

Finally, translation of these experimental data to the human situation might be limited as parameters such as susceptibility to infections and the relation between the dimensions of colon and collagen fleece may apply. It could also be true that in a relatively small animal model such as this, the manipulations needed for application of the fleece induce more trauma than in a larger model. Therefore, we feel that further evaluation, for instance in a sufficiently powered pig model, is mandatory before routine application in a clinical setting.

In conclusion, collagen fleeces kept in place by fibrin glue did not improve bursting pressures in colonic anastomoses, but did increase colonic obstructions in rats. We consider these obstructions an unexpected finding that warrants further evaluation before this product is to be routinely used for the reinforcement of colonic anastomoses in a clinical setting.

Acknowledgements The authors would like to acknowledge J.P.M. Cleutjens, $\mathrm{PhD}$, for his help in analyzing collagen contents.

Disclosures None. Tissucol and Tissufleece were kindly and unconditionally supplied by their manufacturer.

Open Access This article is distributed under the terms of the Creative Commons Attribution Noncommercial License which permits any noncommercial use, distribution, and reproduction in any medium, provided the original author(s) and source are credited.

\section{References}

1. Sharma A, Sharp DM, Walker LG, Monson JR (2007) Predictors of early postoperative quality of life after elective resection for colorectal cancer. Ann Surg Oncol 14(12):3435-3442

2. Urso E, Serpentini S, Pucciarelli S, De Salvo GL, Friso ML, Fabris G, Lonardi S, Ferraro B, Bruttocao A, Aschele C, Nitti D (2006) Complications, functional outcome and quality of life after intensive preoperative chemoradiotherapy for rectal cancer. Eur $\mathbf{J}$ Surg Oncol 32(10):1201-1208

3. Peeters KC, Tollenaar RA, Marijnen CA, Klein Kranenbarg E, Steup WH, Wiggers T, Rutten HJ, van de Velde CJ (2005) Risk factors for anastomotic failure after total mesorectal excision of rectal cancer. Br J Surg 92(2):211-216

4. Kube R, Mroczkowski P, Granowski D, Benedix F, Sahm M, Schmidt U, Gastinger I, Lippert H (2009) Anastomotic leakage after colon cancer surgery: a predictor of significant morbidity and hospital mortality, and diminished tumour-free survival. Eur J Surg Oncol 36:120-124

5. Ptok H, Marusch F, Meyer F, Schubert D, Gastinger I, Lippert H (2007) Impact of anastomotic leakage on oncological outcome after rectal cancer resection. Br J Surg 94(12):1548-1554

6. Enestvedt CK, Thompson SK, Chang EY, Jobe BA (2006) Clinical review: healing in gastrointestinal anastomoses, part II. Microsurgery 26(3):137-143

7. Zacharakis E, Demetriades H, Kanellos D, Sapidis N, Mantzoros I, Kanellos I, Koliakos G, Zaraboukas T, Topouridou K, Betsis D (2007) Contribution of insulin-like growth factor I to the healing of colonic anastomoses in rats. J Invest Surg 20 (1):9-14

8. Kiyama T, Onda M, Tokunaga A, Efron DT, Barbul A (2001) Effect of matrix metalloproteinase inhibition on colonic anastomotic healing in rats. J Gastrointest Surg 5(3):303-311

9. Vignali A, Gianotti L, Braga M, Radaelli G, Malvezzi L, Di Carlo V (2000) Altered microperfusion at the rectal stump is predictive for rectal anastomotic leak. Dis Colon Rectum 43 (1):76-82

10. Jackson MR (2001) Fibrin sealants in surgical practice: an overview. Am J Surg 182(2 Suppl):1s-7s

11. Mutter D, Aprahamian M, Damge C, Sonzini P, Marescaux J (1996) Biomaterial supports for colonic wall defect healing. An experimental study in the rat. Biomaterials 17(14):1411-1415

12. Nordentoft T, Romer J, Sorensen M (2007) Sealing of gastrointestinal anastomoses with a fibrin glue-coated collagen patch: a safety study. J Invest Surg 20(6):363-369

13. Li Y, Bao Y, Jiang T, Tan L, Liu F, Li J (2007) Combination of fibrin glue with growth hormone augments healing of incomplete intestinal anastomoses in a rat model of intra-abdominal sepsis: a dynamic study. J Invest Surg 20(5):301-306

14. van der Ham AC, Kort WJ, Weijma IM, Jeekel H (1993) Transient protection of incomplete colonic anastomoses with fibrin sealant: an experimental study in the rat. J Surg Res 55 (3):256-260

15. Bloemen JG, Schreinemacher MH, de Bruine AP, Buurman WA, Bouvy ND, Dejong CH (2010) Butyrate enemas improve intestinal anastomotic strength in a rat model. Dis Colon Rectum 53(7):1069-1075

16. Christensen H, Chemnitz J, Christensen BC, Oxlund H (1995) Collagen structural organization of healing colonic anastomoses and the effect of growth hormone treatment. Dis Colon Rectum 38 (11):1200-1205

17. Singer AJ, Clark RA (1999) Cutaneous wound healing. N Engl J Med 341(10):738-746 
18. Martens MF, Hendriks T (1991) Postoperative changes in collagen synthesis in intestinal anastomoses of the rat: differences between small and large bowel. Gut 32(12):1482-1487

19. Kanellos I, Mantzoros I, Demetriades H, Kalfadis S, Kelpis T, Sakkas L, Betsis D (2004) Healing of colon anastomoses covered with fibrin glue after immediate postoperative intraperitoneal administration of 5-fluorouracil. Dis Colon Rectum 47(4):510-515

20. Stumpf M, Junge K, Rosch R, Krones C, Klinge U, Schumpelick V (2009) Suture-free small bowel anastomoses using collagen fleece covered with fibrin glue in pigs. J Invest Surg 22(2):138-147

21. Eryilmaz R, Samuk M, Tortum OB, Akcakaya A, Sahin M, Goksel S (2007) The role of dura mater and free peritoneal graft in the reinforcement of colon anastomosis. J Invest Surg 20(1):15-21

22. Ng KW, Khor HL, Hutmacher DW (2004) In vitro characterization of natural and synthetic dermal matrices cultured with human dermal fibroblasts. Biomaterials 25(14):2807-2818

23. Uludag M, Citgez B, Ozkaya O, Yetkin G, Ozcan O, Polat N, Isgor A (2009) Effects of amniotic membrane on the healing of normal and high-risk colonic anastomoses in rats. Int J Colorectal Dis 24(7):809-817

24. Hoeppner J, Crnogorac V, Marjanovic G, Juttner E, Keck T, Weiser HF, Hopt UT (2009) Small intestinal submucosa for reinforcement of colonic anastomosis. Int $\mathrm{J}$ Colorectal Dis 24 (5):543-550

25. Henne-Bruns D, Kreischer HP, Schmiegelow P, Kremer B (1990) Reinforcement of colon anastomoses with polyglycolic acid mesh: an experimental study. European Surgical Research Europaische chirurgische Forschung 22(4):224-230

26. Ozel SK, Kazez A, Akpolat N (2006) Does a fibrin-collagen patch support early anastomotic healing in the colon? An experimental study. Tech Coloproctol 10(3):233-236

27. Merad F, Hay JM, Fingerhut A, Flamant Y, Molkhou JM, Laborde Y (1998) Omentoplasty in the prevention of anastomotic leakage after colonic or rectal resection: a prospective randomized study in 712 patients. French associations for surgical research. Ann Surg 227 (2):179-186

28. Uludag M, Ozdilli K, Citgez B, Yetkin G, Ipcioglu OM, Ozcan O, Polat N, Kartal A, Torun P, Isgor A (2010) Covering the colon anastomoses with amniotic membrane prevents the negative effects of early intraperitoneal 5 -fu administration on anastomotic healing. Int J Colorectal Dis 25(2):223-232

29. Byrne DJ, Hardy J, Wood RA, McIntosh R, Hopwood D, Cuschieri A (1992) Adverse influence of fibrin sealant on the healing of high-risk sutured colonic anastomoses. J R Coll Surg Edinb 37(6):394-398

30. Huh JW, Kim HR, Kim YJ (2010) Anastomotic leakage after laparoscopic resection of rectal cancer: the impact of fibrin glue. Am J Surg 199(4):435-441

31. Efthimiou E, Al Sabah S, Sampalis JS, Christou NV (2009) Fibrin sealant associated with increased body temperature and leukocytosis after laparoscopic gastric bypass. Surg Obes Relat Dis 6:46-49

32. Silecchia G, Boru CE, Mouiel J, Rossi M, Anselmino M, Morino M, Toppino M, Gaspari A, Gentileschi P, Tacchino R, Basso N (2008) The use of fibrin sealant to prevent major complications following laparoscopic gastric bypass: results of a multicenter, randomized trial. Surg Endosc 22(11):2492-2497

33. Saldana Cortes JA, Larios Arceo F, Prieto Diaz Chavez E, De Buen EP, Gonzalez Mercado S, Alvarez Villasenor AS, Prieto Aldape MR, Fuentes Orozco C, Gonzalez Ojeda A (2009) Role of fibrin glue in the prevention of cervical leakage and strictures after esophageal reconstruction of caustic injury. World J Surg 33(5):986-993

34. Upadhyaya VD, Gopal SC, Gangopadhyaya AN, Gupta DK, Sharma S, Upadyaya A, Kumar V, Pandey A (2007) Role of fibrin glue as a sealant to esophageal anastomosis in cases of congenital esophageal atresia with tracheoesophageal fistula. World J Surg 31 (12):2412-2415

35. Jahnson S, Holtz A, Gerdin B (1998) Anastomotic blood-flow reduction in rat small intestine with chronic radiation damage. Digestion 59(2):134-141

36. Egger B, Inglin R, Zeeh J, Dirsch O, Huang Y, Buchler MW (2001) Insulin-like growth factor $i$ and truncated keratinocyte growth factor accelerate healing of left-sided colonic anastomoses. Br J Surg 88(1):90-98

37. Mahdy T, Mohamed G, Elhawary A (2008) A light and scanning electron microscope study of the albino rat ileum after partial obstruction. Annals of anatomy=Anatomischer Anzeiger: official organ of the Anatomische Gesellschaft 190 (6):531-540 